\title{
Balls in Boxes: Variations on a Theme of Warren Ewens and Herbert Wilf
}

\author{
Shalosh B. EKHAD and Doron ZEILBERGER ${ }^{1}$
}

To Herbert Saul Wilf (b. June 13, 1931), on his 80-th birthday

\section{Preface}

There are $r$ boys and $n$ girls. Each boy must pick one girl to invite to be his date in the prom. Although each girl expects to get $R:=r / n$ invitations, most likely, many of them would receive less, and many of them would receive more. Suppose that Nilini, the most "popular" girl, got as many as $m+1$ prom-invitations, is she indeed so popular, or did she just "luck-out"?

Each one of $r$ students has to choose from $n$ different parallel Calculus sections, taught by different professors. Although each professor expects to get $R:=r / n$ students signing-up, most likely, many of them would receive less, and many of them would receive more. Suppose that Prof. Niles, the most "popular" professor got as many as $m+1$ students, is Prof. Niles justified in assuming that she is more popular than her peers, or did she just "luck-out"?

It is Saturday night, and there are $r$ people who have to decide where to dine, and they have $n$ restaurants to choose from. Although each restaurant expects to get $R:=r / n$ diners, most likely, many of them would receive less, and many of them would receive more. Suppose that the Nevada Diner, the most "popular" restaurant, got as many as $m+1$ diners, can they congratulate themselves for the quality of their food, or ambiance, or location, or can they only congratulate themselves for being lucky?

Each one of $r$ cases of acute lymphocitic leukemia has to choose one of $n$ towns (artificially made all with equal-populations) where to happen. Although each town expects to get $R:=r / n$ cases, most likely, many of them would receive less, and many of them would receive more. Suppose that the Illinois town Niles had $m+1$ cases of that disease, do its people have to be concerned about their environment, or is it only Lady Luck's fault?

Of course all these questions have the same answer, and typically one talks about $r$ balls being placed, uniformly at random, in $n$ boxes, where the largest number of balls that landed at the same box was $m+1$. Yet another way: A monkey is typing an $r$-letter word using a keyboard of an alphabet with $n$ letters, and the most frequent letter showed-up $m+1$ times. Does the typing

1 Department of Mathematics, Rutgers University (New Brunswick), Hill Center-Busch Campus, 110 Frelinghuysen Rd., Piscataway, NJ 08854-8019, USA. zeilberg at math dot rutgers dot edu ,

http://www.math.rutgers.edu/ zeilberg/. Written: June 13, 2011. Accompanied by Maple package

http://www.math.rutgers.edu/ ${ }^{\sim}$ zeilberg/tokhniot/BallsInBoxes. Sample input and output can be gotten from: http://www.math.rutgers.edu/ zeilberg/mamarim/mamarimhtml/bib.html .

Supported in part by the National Science Foundation of the United States of America. 
monkey have a particular fondness for that letter, or is he a truly uniformly-at-random monkey who does not play favorites with the letters?

\section{Asking the Right Question}

As Herb Wilf pointed out so eloquently in his wonderful talk at the conference W80 (celebrating his 80th birthday) (based, in part, on [EW]), using the depressing disease formulation, the right questions are not:

"What is the probability that Nilini would get so many ( $m+1$ of them) prom-invitations?"

"What is the probability that Prof. Niles would get so many $(m+1$ of them) students?"

"What is the probability that the Nevada Diner would get so many ( $m+1$ of them) diners?"

"What is the probability that Niles, IL would get so many ( $m+1$ of them) cases of acute lymphocitic leukemia?"

Even though this is the wrong question (whose answer would make Nilini, Prof. Niles and the Nevada Diner's successes go to their heads, and would make the real-estate prices in Niles, IL, plummet), because it is so tiny, and seemingly extremely unlikely to be "due to chance", let's answer this question anyway.

The a priori probability of Nilini getting $m+1$ or more prom-invitations, using the Poisson Approximation is:

$$
e^{-R}\left(\sum_{i=m+1}^{\infty} \frac{R^{i}}{i !}\right)=e^{-R}\left(e^{R}-\sum_{i=0}^{m} \frac{R^{i}}{i !}\right)=1-e^{-R} \sum_{i=0}^{m} \frac{R^{i}}{i !},
$$

indeed very small if $m$ is considerably larger than $R$.

But a priori we don't know who would be the "lucky champion" (or the unlucky town), the right question to ask is:

The Right Question: Given $r, n$, and $m$, compute (if possible exactly, but at least approximately):

$P(r, n, m):=$ : the probability that every box got $\leq m$ balls.

\section{Getting the Right Answer to the Right Question, as Fast as Possible}

In [EW], Ewens and Wilf present a beautiful, fast $(O(m n))$, algorithm for computing the exact value of $P(r, n, m)$, that employs a method that is described in the Nijenhuis-Wilf classic [NW] (but that has been around for a long time, and rediscovered several times, e.g. by one of us ([Z1]), and before that by J.C.P. Miller, and according to Don Knuth the method goes back to Euler. At any rate, $[\mathrm{EW}]$ does not claim novelty for the method, only for applying it to the present problem).

The specific real-life examples given in $[\mathrm{EW}]$ were: 
1. (Niles, IL): $r=14400, n=9000$, (so $R=8 / 5$ ), $m=7$. Using their method, they got (in less than one second!) the value

$$
P(14400,9000,7)=0.0953959131671303999971555481626 \ldots \quad,
$$

meaning that the probability that every town in the US, of the size of Niles, IL, would get no more than 7 cases is less than ten percent. So with probability 0.904604086832869600002844451837 , some town (of the same size, assuming, artificially that the US has been divided into towns of that size) somewhere, in the US, would get at least eight cases. There is (most probably) nothing wrong with their water, or their air-quality, the only one that they may blame is Lady Luck!

For comparison, the a priori probability that Niles,IL would get 8 or more cases is roughly:

$$
1-e^{-1.6} \sum_{i=0}^{7} \frac{1.6^{i}}{i !}=0.00026044 \ldots,
$$

a real reason for (unjustified!) concern.

2. (Churchill County, NV): $r=8000, n=12000$, (so $R=2 / 3$ ), $m=11$. Using their method, they got (in less than one second!) the value

$$
P(8000,12000,11)=0.999999895529647647310726013392 \ldots \quad,
$$

so it is extremely likely that every district got at most 11 cases, and the probability that some district got 12 or more cases is indeed small, namely

$$
1-P(8000,12000,11)=0.104470 \cdot 10^{-6},
$$

so these people should indeed panic.

For comparison, the a priori probability that Churchill County, NV, would get 12 or more cases is roughly:

$$
1-e^{-2 / 3} \sum_{i=0}^{11} \frac{(2 / 3)^{i}}{i !}=.870586315 \cdot 10^{-11},
$$

in that case people would have been right to be concerned, but for the wrong reason!

\section{The Maple package BallsInBoxes}

This article is accompanied by the Maple package BallsInBoxes available from: http://www . math.rutgers.edu/〜zeilberg/tokhniot/BallsInBoxes .

Lots of sample input and output files can be gotten from: http://www . math.rutgers. edu/ zeilberg/mamarim/mamarimhtml/bib.html . 


\section{How to Compute $P(r, n, m)$ Exactly?}

Easy! As Ewens and Wilf point out in [EW], and Herb Wilf mentioned in his talk, there is an obvious, explicit, "answer"

$$
P(r, n, m)=\frac{1}{n^{r}} \sum \frac{r !}{r_{1} ! r_{2} ! \ldots r_{n} !},
$$

where the sum ranges over the set of $n$-tuples of integers

$$
A(r, n, m):=\left\{\left(r_{1}, r_{2}, \ldots, r_{n}\right) \mid 0 \leq r_{1}, \ldots, r_{n} \leq m \quad, \quad r_{1}+r_{2}+\ldots+r_{n}=r\right\} \quad .
$$

So "all" we need, in order to get the exact answer, is to construct the set $A(r, n, m)$ and add-up all the multinomial coefficients.

Of course, there is a better way. As is well-known (see [EW]), and easy to see, writing

$$
P(r, n, m)=\frac{r !}{n^{r}} \sum_{\left(r_{1}, \ldots, r_{n}\right) \in A(r, n, m)} \frac{1}{r_{1} ! r_{2} ! \ldots r_{n} !}
$$

the $\sum$ is the coefficient of $x^{r}$ in the expansion of

$$
\left(\sum_{i=0}^{m} \frac{x^{i}}{i !}\right)^{n}
$$

so all we need is to go to Maple, and type (once $r, n$, and $m$ have been assigned numerical values)

$\mathrm{r} ! / \mathrm{n} * * \mathrm{r} * \operatorname{coeff}(\operatorname{add}(\mathrm{x} * * i / \mathrm{i} !, \mathrm{i}=0 \ldots \mathrm{m}) * * \mathrm{n}, \mathrm{x}, \mathrm{r}) ; \quad$.

This works well for small $n$ and $r$, but, please, don't even try to apply it to the first case of [EW], $(r=14400, n=9000, m=7)$, Maple would crash!

Ewens and Wilf's brilliant idea was to use the Euler-Miller-(Nijenhuis-Wilf)-Zeilberger-... "quick" method for expanding a power of a polynomial, and get an answer in less than a second!

[We implemented this method in Procedure Prnm $(r, n, m)$ of BallsInBoxes].

While their method indeed takes less than a second (in Maple) for $r=14400, n=9000$ (and $7 \leq m \leq 12$ ), it takes quite a bit longer for $r=144000, n=90000$, and I am willing to bet that for $r=10^{8}, n=10^{8}$ it would be hopeless to get an exact answer, even with this fast algorithm.

But why this obsession with exact answers? Hello, this is applied mathematics, and the epidemiological data is, of course, approximate to begin with, and we make lots of unrealistic assumptions (e.g. that the US is divided into 9000 towns, each exactly the size of Niles, IL.) . All we need to know is, "are that many diseases likely to be due to pure chance, or is it a cause for concern", Yes or No?, Ja oder Nein?, Oui ou Non?, Ken o Lo?. 


\section{Enumeration Digression}

It would be nice to get a more compact (than the huge multisum above) (symbolic) "answer", or "formula", in terms of the symbols $r, n$ and $m$. This seems to be hopeless. But fixing, positive integers $a, b$ and $m$, one can ask for a "formula" (or whatever), in $n$, for the quantity $P(a n, b n, m)$ that can be written as $B(a, b, m ; n) /(a n)^{b n}$ where

$$
B(a, b, m ; n):=(a n) ! \sum_{\left(r_{1}, \ldots, r_{n}\right) \in A(a n, b n ; m)} \frac{1}{r_{1} ! r_{2} ! \ldots r_{n} !}
$$

the cardinality of the natural combinatorial set consisting of placing an balls in $b n$ boxes in such a way that no box receives more than $m$ balls. Equivalently, all words in a bn-letter alphabet, of length $a n$, where no letter occurs more than $m$ times. For example, when $a=b=m=1$, we have the deep theorem:

$$
B(1,1,1 ; n)=n !
$$

Equivalently, $e(n)=B(1,1,1 ; n)$ is a solution of the linear recurrence equation with polynomial coefficients

$$
e(n+1)-(n+1) e(n)=0 \quad, \quad(n \geq 0),
$$

subject to the initial condition $e(0)=1$.

It turns out that, thanks to the not-as-famous-as-it-should-be Almkvist-Zeilberger algorithm [AZ] (an important component of the deservedly famous Wilf-Zeilberger Algorithmic Proof Theory), one

can find similar recurrences (albeit of higher order, so it is no longer "closed-form", in $n$ ) for the sequences $B(a, b, m ; n)$ for any fixed triple of positive integers, $a, b, m$.

(See Procedures Recabm and RacabmV in the Maple package BallsInBoxes).

Indeed, since $B(a, b, m ; n)$ is $(a n)$ ! times the coefficient of $x^{a n}$ in

$$
\left(\sum_{i=0}^{m} \frac{x^{i}}{i !}\right)^{b n}
$$

it can be expressed, (thanks to Cauchy), as

$$
\frac{(a n) !}{2 \pi i} \oint_{|z|=1} \frac{\left(\sum_{i=0}^{m} \frac{z^{i}}{i !}\right)^{b n}}{z^{a n+1}} d z
$$

and this is game for the Almkvist-Zeilberger algorithm, that has been incorporated into BallsInBoxes. See the web-book http://www.math.rutgers.edu/ zeilberg/tokhniot/oBallsInBoxes2

for these recurrences for $1 \leq a, b \leq 3$ and $1 \leq m \leq 6$. 


\section{Asymptotics}

Once the first-named author of the present article computed a recurrence, it can go on, thanks to the Birkhoff-Trzcinski method ([WimZ][Z2]), to get very good asymptotics! So now we can get a very precise asymptotic formula (in $n$ ) (to any desired order!) for $P(a n, b n, m)$, that turns out to be very good for large, and even not-so-large $n$, and for any desired $a, b, m$. Procedure Asyabm in our Maple package BallsInBoxes finds such asymptotic formulas. See

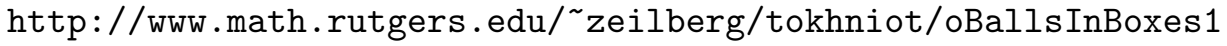

for asymptotic formulas, derived by combining Almkvist-Zeilberger with AsyRec (also included in BallsInBoxes in order to make the latter self-contained.)

This works for every $m$, and every $a$ and $b$, in principle! In practice, as $m$ gets larger than 10, the recurrences become very high order, and take a very long time to derive.

But as long as $m \leq 8$ and even (in fact, especially) when $n$ is very large, this method is much faster than the method of [EW] $(O(m n)$ with large $n$ is not that small!). Granted, it does not give you an exact answer, but neither do they (in spite of their claim, see below!) .

But let's be pragmatic and forget about our purity and obsession with "exact" answers. Since we know from "general nonsense" that the asymptotics of the desired probability

$$
C(a, b, m ; n):=P(a n, b n, m) \quad\left(=B(a, b, m ; n) /(a n)^{b n}\right)
$$

behaves asymptotically as

$$
C(a, b, m ; n) \asymp \mu^{n}\left(c_{0}+O(1 / n)\right) \quad,
$$

for some numbers $\mu$ and $c_{0}$, all we have to do is crank out (e.g.) the 200-th and 201-th term and estimate $\mu$ to be $C(a, b, m ; 201) / C(a, b, m ; 200)$, and then estimate $c_{0}$ to be $C(a, b, m ; 200) / \mu^{200}$. Using Least Squares one can do even better, and also estimate higher order asymptotics (but we don't bother, enough is enough!).

Procedure AsyabmEmpir in our Maple package BallsInBoxes uses this method, and gets very good results!

For example, for the Niles, IL, example, in order to get estimates for $P(14400,9000, m)$, typing

evalf (subs (n=1800, AsyabmEmpir $(8,5, m, 200, n)))$;

for $m=7,8,9,10,11,12$ yields (almost instantaneously)

$m=7: 0.09540287131 \ldots$ (the exact value being: $0.095395913167 \ldots)$,

$m=8: 0.664971462304 \ldots$ (the exact value being: $0.66495441 \ldots)$,

$m=9: 0.9378712268719 \ldots$ (the exact value being: $0.93786433 \ldots$ ) 
$m=10: 0.990845139 \ldots$ (the exact value being: $0.9908433 \ldots)$

$m=11: 0.998789295 \ldots$ (the exact value being: $0.99878892861 \ldots)$.

The advantage of the present approach is that we can handle very large $n$, for example, with the same effort we can compute

evalf (subs ( $\mathrm{n}=180000$, AsyabmEmpir $(8,5, \mathrm{~m}, 200, \mathrm{n})))$

getting, for example, that $P(1440000,900000,11)$ is very close to 0.88554890636027 . The method used in [EW] (i.e. typing Prnm $(1440000,900000,11)$; in BallsInBoxes) would take forever!

\section{Caveat Emptor}

There is another problem with the $O(m n)$ method described in [EW]. Sure enough, it works well for the examples given there, namely $P(14400,9000, m)$ for $6 \leq m \leq 12$ and $P(8000,12000, m)$ for $4 \leq m \leq 8$.

This is corroborated by our implementation of that method, (Procedure Prnm $(r, n, m)$ in BallsInBoxes).

Typing (once BallsInBoxes has been read onto a Maple session):

t0:=time(): Prnm(14400,9000,9), time()-t0;

returns

$0.937864339305858219725360911354,0.884$

that tells you the desired value (we set Digits to be 30 ), and that it took 0.884 seconds to compute that value.

But now try:

t0:=time(): Prnm(1000,100,15), time()-t0;

and get in 0.108 seconds (real fast!)

$-0.728465229161818857989128673465 \cdot 10^{50}$.

Something is rotten in Denmark! We learned in kindergarten that a probability has to be between 0 and 1 , so a negative probability, especially one with 50 decimal digits, is a bit fishy. Of course, the problem is that [EW]'s "exact" result is not really exact, as it uses floating-point arithmetic.

Big deal, since we work in Maple, let's increase the system variable Digits (the number of digits used in floating-point calculations), and type the following line: 
evalf (Prnm $(1000,100,15), 80)$;

getting $5.71860506564981 \ldots$, a little bit better! (the probability is now less than six, and at least it is positive!), but still nonsense.

Digits:=83 still gives you nonsense, and it only starts to "behave" at Digits: $=90$.

Now let's multiply the inputs, $r$ and $n$ by 10, and take $m=22$ and try to evaluate $P(10000,1000,22)$. Even Digits: $=250$ still gives nonsense! Only Digits:=310 gives you something reasonable and (hopefully) correct.

The way to overcome this problem is to keep upping Digits until you get close answers with both Digits and, say, Digits+100. This is implemented in Procedure

PrnmReliable $(r, n, m, k)$ in BallsInBoxes, if one desires an accuracy of $k$ decimal digits. This is reliable indeed, but not exact, and not rigorous, since it uses numerical heuristics. The exact answer is a rational number, that is implemented in Procedure PrnmExact $(r, n, m)$ of BallsInBoxes.

\section{The Cost of Exactness}

If you type

to:=time ():PrnmExact $(14400,9000,7):$ time()-t0;

you would get in 42 seconds (no longer that fast!) a rational number whose numerator and denominator are exact integers with 54207 digits.

See

http://www . math.rutgers.edu/ zeilberg/tokhniot/oBallsInBoxes7a

for the outputs (and timings) of PrnmExact $(14400,9000, \mathrm{~m})$; for $m$ between 6 and 12 and see http://www .math.rutgers.edu/ ${ }^{\sim}$ zeilberg/tokhniot/oBallsInBoxes7b

for the outputs (and timings) of PrnmExact $(8000,12000, \mathrm{~m})$; for $m$ between 4 and 8 . No longer fast at all! (2535 and 248 seconds respectively).

\section{Let's Keep It Simple: An Ode to the Poisson Approximation}

At the end of $[\mathrm{EW}]$, the authors state:

"A Poisson Approximation is also possible but it may be inaccurate, particularly around the tails of the distribution. Our exact method is fast and does not suffer from any of those problems."

Being curious, we tried it out, to see if it is indeed so bad. Surprise, it is terrific! But let's first review the Poisson approximation as we understand it. 
The probability of any particular box (of the $n$ boxes) getting $\leq m$ ball is, roughly, using the Poisson approximation $(R:=r / n)$ :

$$
e^{-R} \sum_{i=0}^{m} \frac{R^{i}}{i !} .
$$

Of course the $n$ events are not independent, but let's pretend that they are. The probability that every box got $\leq m$ balls is approximated by

$$
Q(r, n, m):=\left(e^{-R} \sum_{i=0}^{m} \frac{R^{i}}{i !}\right)^{n} .
$$

$[Q(r, n, m)$ is implemented by procedure $\operatorname{PrnmPA}(\mathrm{r}, \mathrm{n}, \mathrm{m})$ in BallsInBoxes. It is as fast as lightning!]

Ewens and Wilf are very right when they claim that $P(r, n, m)$ and $Q(r, n, m)$ are very far apart around the "tail" of the distribution, but who cares about the tail? Definitely not a scientist and even not an applied mathematician. It turns out, empirically (and we did extensive numerical testing, see Procedure HowGoodPA1 (R0, NO, Incr, MO, m, eps) in BallsInBoxes), that whenever $P(r, n, m)$ is not extremely small, it is very well approximated by $Q(r, n, m)$, and using the latter (it is so much faster!) gives very good approximations, and enables one to construct the "center" of the probability distribution (i.e. ignoring the tails) very accurately. See

http://www.math.rutgers.edu/ zeilberg/tokhniot/oBallsInBoxes4,

and

http://www.math.rutgers.edu/ zeilberg/tokhniot/oBallsInBoxes5, ,

for comparisons (and timings!, the Poisson Approximation wins!) .

In particular, the estimates for the expectation, standard deviation, and even the higher moments match extremely well!

Another (empirical!) proof of the fitness of the Poisson Approximation can be seen in:

http://www.math.rutgers.edu/ zeilberg/tokhniot/oBallsInBoxes1

where the (rigorous!) asymptotic formulas derived, via AsyRec, from the recurrences obtained via the Almkvist-Zeilberger algorithm are very close to those predicted by the Poisson Approximation (except for very small $m$, corresponding to the "tail").

\section{The Full Probability Distribution of the Random Variable "Maximum Number of Balls in the Same Box"}

It would be useful, for given positive integers $a$ and $b$, to know how the probability distribution "maximum number of balls in the same box when throwing an balls into bn boxes" behaves. One can "empirically" construct (without arbitrarily improbable tail) the distribution of the random 
variable "maximum number of balls in the same box" when an balls are uniformly-at-random placed in $b n$ boxes (Let's call it $X_{n}(a, b)$, and $X_{n}$ for short) using

$$
\operatorname{Pr}\left(X_{n}=m\right)=P(a n, b n, m)-P(a n, b n, m-1) .
$$

First, and foremost, what is the expectation, $\mu_{n}$ ? Second what is the standard deviation, $\sigma_{n}$ ?, skewness?, kurtosis?, and it would ve even nice to know higher $\alpha$-coefficients (alias moments of $\left.Z_{n}:=\left(X_{n}-\mu_{n}\right) / \sigma_{n}\right)$, as asymptotic formulas in $n$.

For the expectation, $\mu_{n}$, Procedure AveFormula ( $\left.\mathrm{a}, \mathrm{b}, \mathrm{n}, \mathrm{d}, \mathrm{L}, \mathrm{k}\right)$ uses the more accurate "empirical approach" and Maple's built-in Least-Squares command, to obtain the following empirical (symbolic!) estimates for the expectation.

$a=1, b=1$ : evalf (AveFormula $(1,1, \mathrm{n}, 1,300,1000,10), 10)$; yields that

$\mu_{n}$ is roughly $2.293850526+(0.4735983525) \cdot \log n$

$a=2, b=1$ : evalf (AveFormula $(2,1, \mathrm{n}, 1,300,1000,10), 10)$; yields that

$\mu_{n}$ is roughly $3.963420618+(0.5834252496) \cdot \log n$

$a=1, b=2$ : evalf (AveFormula $(1,2, \mathrm{n}, 1,300,1000,10), 10)$; yields that

$\mu_{n}$ is roughly $1.640094145+(0.3873602232) \cdot \log n$.

Note that for $a=1, b=1$, the approximation to $\mu_{n}$ can be written

$2.293850526+(1.090500507) \cdot \log _{10} n$, so a "rule-of-thumb" estimate for the expectation when $n$ balls are thrown into $n$ boxes is a bit more than 2 plus the number of (decimal) digits.

Procedure NuskhaPA1 $(\mathrm{R}, \mathrm{n}, \mathrm{K}, \mathrm{d})$ uses the Poisson Approximation to guess polynomials in $\log n$ of degree $d$ fitting the average, standard deviation, and higher moments, as asymptotic expressions in $n$, for $n R$ balls thrown into $n$ boxes, where $R$ is now any (numeric) rational number. Even $d=1$ seems to give a fairly good fit, so they all seem to be (roughly) linear in $\log n$.

\section{Procedure SmallestmPA}

Procedure SmallestmPA ( $r, \mathrm{n}, \mathrm{conf})$ gives you the smallest $m$ for which, with confidence conf, you can deduce that the high value of $m$ is not due to chance (using the Poisson Approximation). For example

SmallestmPA $(14400,9000, .99)$;

yields 10, meaning that if a town the size of Niles, IL got 10 or more cases, then with probability $>0.99$ it is not just bad luck. If you want to be \%99.99-sure of being a victim of the environment rather than of Lady Luck, type:

SmallestmPA (14400, 9000, .9999); 
and get 13, meaning that if you had 13 cases, then with probability larger than 0.9999 it is not due to chance.

\section{The Minimum Number of Balls that Landed in the Same Box, Procedure LargestmPA}

An equally interesting, and harder to compute, random variable is the minimum number of balls that landed in the same box, but the Poisson Approximation handles it equally well. Analogous to SmallestmPA, we have, in BallsInBoxes, Procedure LargestmPA ( $r, n$, conf) that tells you the largest $m$ for which you can't blame luck for getting $m$ or less balls.

For example, if there are 10000 students that have to decide between 100 different calculus sections,

LargestmPA $(10000,100, .99)$;

that happens to be 66 , tells you that any section that only has 66 students or less, with probability $>0.99$, it is because that professor (or time slot, e.g. if it is an 8:00am class) is not popular, and you can't blame bad luck.

LargestmPA $(10000,100, .9999)$;

that outputs 57, tells you that anyone who only had $\leq 57$ students enrolled is unpopular with probability $>\% 99.99$, and can’t blame bad luck.

On the other end, going back to the original problem,

SmallestmPA $(10000,100, .99)$; yields 139 , telling you that any section for which 139 or more students signed up is probably (with prob. $>0.99$ ) due to the popularity of that section, while SmallestmPA (10000,100, .9999); yields 151.

\section{Final Comments}

1. One can possibly (using the saddle-point method) get asymptotic formulas from the contour integral (Cauchy), but this is not our cup-of-tea, so we leave it to other people.

2. Another "back-of-the-envelope" "Poisson Approximation" is to argue that since the probability of any individual box getting strictly more than $m$ balls is roughly (recall that $R=r / n$ )

$$
e^{-R} \sum_{i=m+1}^{\infty} \frac{R^{i}}{i !}=e^{-R}\left(e^{R}-\sum_{i=0}^{m} \frac{R^{i}}{i !}\right)=1-e^{-R} \sum_{i=0}^{m} \frac{R^{i}}{i !},
$$

by the linearity of expectation, the expected number of lucky (or unlucky if the balls are diseases) boxes exceeding $m$ balls is roughly

$$
n\left(1-e^{-R} \sum_{i=0}^{m} \frac{R^{i}}{i !}\right) .
$$

In the case of Niles, IL, the expected number of towns that would get 8 or more cases is:

$$
9000\left(1-e^{-1.6} \sum_{i=0}^{7} \frac{(1.6)^{i}}{i !}\right)=2.343961376410372,
$$


so it is not at all surprising that at least one town got as many as 8 cases. On the other hand, in the other example $r=8000, n=12000, m=12$, the expected number of unfortunate counties is:

$$
12000\left(1-e^{-(2 / 3)} \sum_{i=0}^{12} \frac{(2 / 3)^{i}}{i !}\right)=0.533706802 \cdot 10^{-8},
$$

so it is indeed a reason for concern.

\section{Conclusion}

We completely agree with Ewens and Wilf that Monte Carlo takes way too long, and is not that accurate, and that their method is far superior to it. But we strongly disagree with their dismissal of the Poisson Approximation. In fact, we used their ingenious method to conduct extensive empirical (numerical) testing that established that the Poisson Approximation, that they dismissed as "inaccurate", is, as a matter of fact, sufficiently accurate, far more reliable, in addition to being yet-much-faster! It is much safer to use the Poisson Approximation than to use their "exact" method (in floating-point arithmetic), and when one uses truly exact calculations, in rational arithmetic, their "fast" method becomes anything but.

Even when the floating-point problem is addressed by using multiple precision (PrnmReliable discussed above), their fast algorithm becomes slow for very large $r$ and $n$, while the Poisson Approximation is almost instantaneous even for very large $r$ and $n$, and any $m$.

So while we believe that the algorithm in $[\mathrm{EW}]$ is not as useful as the Poisson Approximation, it sure was meta-useful, since it enabled us to conduct extensive numerical testing that showed, once and for all, that it is far less useful then the latter.

Additional evidence comes from our own symbolic approach (fully rigorous for $m \leq 9$ and semirigorous for higher values of $m$ ), that establishes the adequacy of the Poisson Approximation for symbolic $n$.

Finally, as we have already pointed out, since the data that one gets in applications is always approximate to begin with, insisting on an "exact" answer, even when it is easy to compute, is unnecessary.

\section{Coda: But We, Enumerators, Do Care About Exact Results!}

Our point, in this article, was that for applications to statistics, the Poisson Approximation suffices. But we are not statisticians. We are enumerators, and we do like exact results! The approach of [EW] enables us to know, for example, in less than one second the exact number of ways that 1001 balls can be placed in 1001 boxes such that no box received more than 7 balls. Just type (in BallsInBoxes)

$(1001 * * 1001) * \operatorname{PrnmExact}(1001,1001,7)$;

and get a beautiful exact integer with 3004 digits! 
Typing

$(1001 * * 1001) * \operatorname{PrnmPA}(1001,1001,7)$;

will give you something fairly close (the ratio being $0.9997852 \ldots$. .)

but for a pure enumerator, this is very unsatisfactory. So long live exact answers!, but not in statistics.

\section{References}

[AZ] Gert Almkvist and Doron Zeilberger, The Method of Differentiating Under The Integral Sign, J. Symbolic Computation 10 (1990), 571-591. [Available on-line from:

http://www .math.rutgers.edu/〜zeilberg/mamarim/mamarimPDF/duis.pdf]

[EW] Warren J. Ewens and Herbert S. Wilf, Computing the distribution of the maximum in ballsand-boxes problems with application to clusters of disease cases, Proc. National Academy of Science (USA) 104(7) (July 3, 2007), 11189-1191 . [Available on-line from:

http://www .pnas.org/content/104/27/11189.full.pdf]

[WimZ] Jet Wimp and Doron Zeilberger, Resurrecting the asymptotics of linear recurrences, J. Math. Anal. Appl. 111 (1985), 162-177. [Available on-line from:

http://www.math.rutgers.edu/ zeilberg/mamarimY/WimpZeilberger1985.pdf]

[Z1] Doron Zeilberger, The J.C.P. Miller Recurrence for exponentiating a polynomial, and its qAnalog, J. Difference Eqs. and Appl. 1 (1995), 57-60. [Available on-line from:

http://www.math.rutgers.edu/ zeilberg/mamarim/mamarimhtml/power.html]

[Z2] Doron Zeilberger AsyRec: A Maple package for Computing the Asymptotics of Solutions of Linear Recurrence Equations with Polynomial Coefficients, The Personal Journal of Shalosh B. Ekhad and Doron Zeilberger http://www.math.rutgers.edu/ zeilberg/pj.html, April 6, 2008. [Article and package available on-line from:

http://www.math.rutgers.edu/ zeilberg/mamarim/mamarimhtml/asy.html] 